\title{
Analgesia para cirugía pediátrica ambulatoria
}

\section{Pain management for pediatric ambulatory surgery}

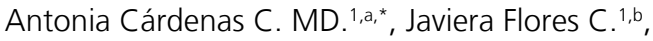 \\ Anestesióloga Clínica Pontificia Universidad Católica de Chile. \\ Anestesióloga Clínica. \\ Interna de Medicina.
}

Financiamiento: ninguno.

Conflicto de intereses: los autores declaran no tener conflicto de intereses.

Fecha de ingreso: 27 de noviembre de 2020 / Fecha de aceptación: 30 de enero de 2021

\begin{abstract}
Introduction: Outpatient pediatric surgery is very frequent. However, sometimes analgesia is insufficient. Implementation of regional anesthesia reduces consume of systemic analgesics, especially opioids. The objective of this article is to review available evidence regarding pain management in pediatric ambulatory surgery, in order to contribute to better care of these patients in this field of medicine. A search was made on Pubmed, ProQuest, and EMBASE databases obtaining a total of 973 articles. The titles of articles found were reviewed, of which 152 merited greater review. Finally, a total of 40 articles to be included in this review were selected. Results: Acetaminophen and nonsteroidal anti-inflammatory drugs are safe in children. Opioid use has decreased to avoid potential adverse events. Advances in ultrasound technology have contributed to safer and efficient peripheral nerve blocks, comparable to neuroaxial techniques, decreasing the use of systemic analgesia, adverse events and allowing early discharge. Conclusion: A multimodal approach handle perioperative pain adequately and minimize perioperative complications. With the use of ultrasound, regional anesthesia is a safe technique that offers multiple advantages in ambulatory surgical procedures, ensuring an early discharge. More evidence is needed to recommend the use of adyuvant drugs and continuos peripheral nerve blocks in children.
\end{abstract}

Key words: Analgesia, pediatrics, ambulatory surgical procedures, pain.

\section{RESUMEN}

Introducción: La cirugía pediátrica es realizada frecuentemente en forma ambulatoria, sin embargo, la analgesia al alta es inadecuada en algunos casos. El uso de analgesia regional ha permitido disminuir el consumo de analgésicos sistémicos, particularmente opioides. El objetivo de este trabajo es revisar la evidencia disponible respecto a técnicas de manejo del dolor pos-operatorio en contexto de cirugía pediátrica ambulatoria, de manera de contribuir al mejor cuidado de estos pacientes en esta área de la medicina. Se realizó una búsqueda en Pubmed, Embase y ProQuest, encontrándose un total de 973 trabajos. Se revisaron los títulos, encontrando 152 de interés para mayor revisión. Se seleccionaron finalmente 40 trabajos para ser incluidos en esta revisión. Resultados: Paracetamol y antiinflamatorios no esteroidales son seguros de usar en niños. El uso de opioides es cada vez menos frecuente dado su perfil de efectos adversos. Con el uso de ecografía los bloqueos de nervio periférico son cada vez más usados, logrando analgesia similar a las técnicas neuroaxiales, disminuyendo el consumo de analgésicos, efectos adversos y logrando un alta precoz. Conclusión: La analgesia multimodal permite un adecuado manejo del dolor, reduciendo los efectos adversos. Gracias a la amplia disponibilidad de ultrasonido, la analgesia regional es cada vez más segura y eficaz, ofreciendo ventajas en el contexto de cirugía ambulatoria al permitir un alta precoz. Es necesaria más evidencia para recomendar el uso de adyuvantes y bloqueos continuos de nervio periférico en contexto de cirugía pediátrica ambulatoria.

Palabras clave: Analgesia, pediatría, procedimientos quirúrgicos ambulatorios, dolor.

antonia.cardenas@gmail.com

*ORCID: https://orcid.org/0000-0001-5146-4532 


\section{Introducción}

a cirugía pediátrica es realizada frecuentemente en forma ambulatoria[1]. La rápida recuperación de los pacientes pediátricos y la ausencia de comorbilidades hacen factible el alta precoz en cirugías de baja-mediana complejidad, reduciendo la ansiedad de separación y los costos asociados a hospitalización[1]. El manejo adecuado del dolor posoperatorio es relevante, buscando una cobertura permanente para evitar las tasas de dolor moderado-severo, descritas en 30\%-70\%[2],[3], el cual puede prolongarse varios días[4]. Esto se ha asociado a mayores costos y consecuencias adversas físicas y psicológicas[1]. Por otro lado, el manejo inadecuado del dolor prolonga la estadía en recuperación y aumenta las hospitalizaciones no programadas[1]

El manejo del dolor posoperatorio se realiza dentro de esquemas de analgesia multimodal, incluyendo el uso de anestésicos locales $(A L)$, analgésicos orales como paracetamol, antiinflamatorios no esteroidales (AINES) u opioides. Estos últimos, si bien proporcionan buena analgesia, dado su perfil de efectos adversos (EA) son cada vez menos usados en contexto ambulatorio[2]. La anestesia regional constituye un pilar fundamental, siendo los bloqueos caudales (BC) los más utilizados para proporcionar analgesia intra y posoperatoria. No obstante, pueden tener riesgos asociados como bloqueo motor y retención urinaria cuando se usan dosis inadecuadas. En los casos que existe contraindicación a las técnicas neuroaxiales, los bloqueos de nervio periférico (BNP) han sido cada vez más usados en pacientes pediátricos en contexto de cirugía ambulatoria[5],[6], aunque la evidencia es limitada.

El objetivo de este trabajo es realizar una revisión narrativa de la evidencia disponible respecto a técnicas de manejo del dolor posoperatorio en cirugía pediátrica ambulatoria, de manera de contribuir al mejor cuidado de estos pacientes.

Para esto se realizó una búsqueda en Pubmed, ProQuest y Embase usando los términos "analgesia", "pediatric", "ambulatory", "surgery", "outpatient" y sus respectivas combinaciones, publicados en los últimos 10 años, encontrandose un total de 973 trabajos. Se seleccionaron por título aquellos de interés resultando un total de 152 trabajos. Posteriormente, se revisaron los resúmenes de dichos trabajos, seleccionando exclusivamente artículos en inglés o español, que no correspondieran a cartas a editor o series de casos, incluyendo revisiones, metaanálisis, estudios clínicos randomizados y estudios observacionales. Además, se realizó una revisión manual de referencias de los trabajos seleccionados y sugerencias que parecieron pertinentes. Finalmente, se incluyeron 40 artículos en esta revisión (Figura 1).

\section{Dolor posoperatorio en cirugía ambulatoria pediátrica}

En Chile, las cirugías pediátricas más frecuentemente realizadas en forma ambulatoria son las cirugías urológicas como circunsiciones u orquidopexia, de pared abdominal como reparaciones de hernias inguinales o umbilicales, oftalmológicas y

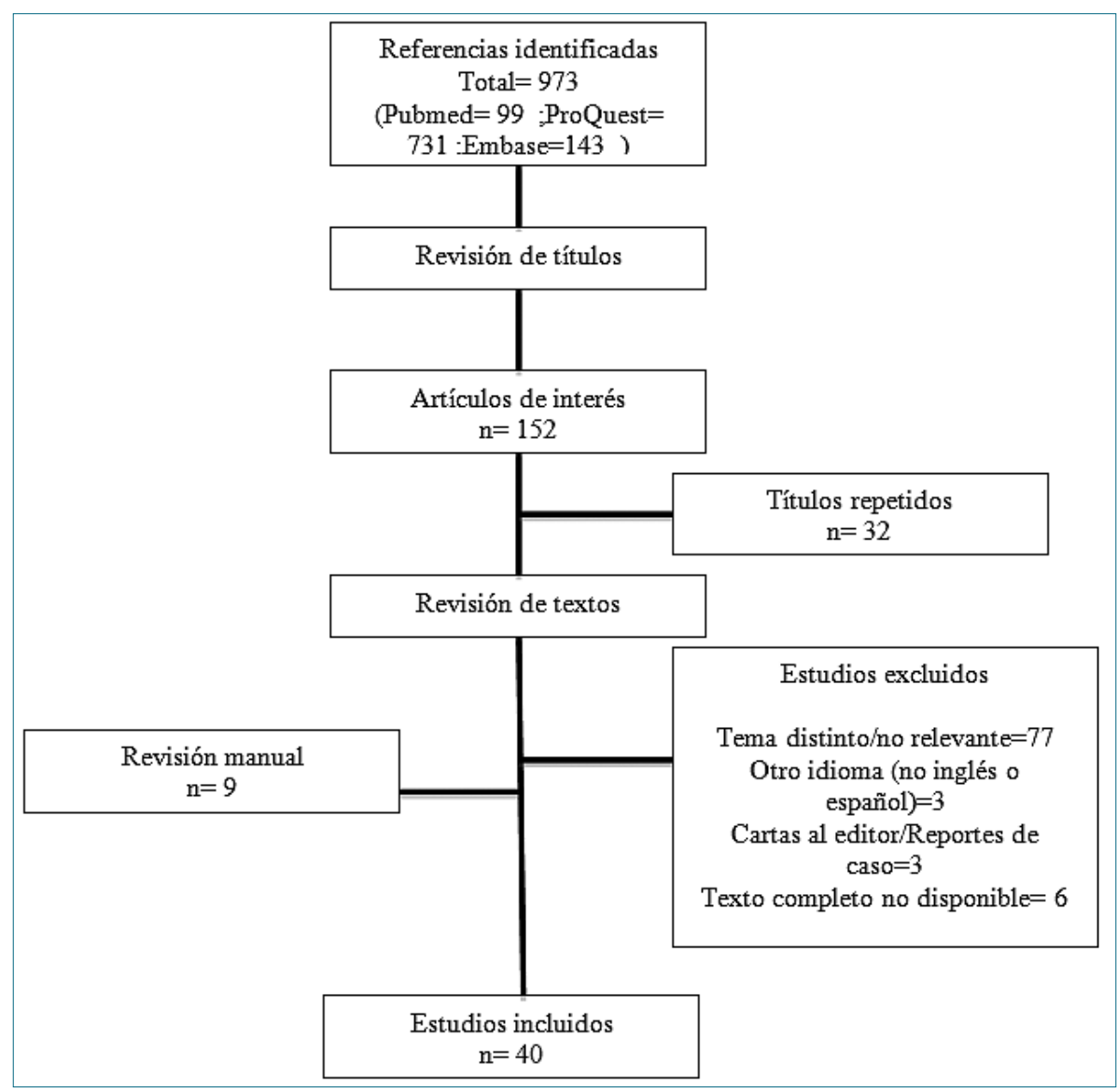

Figura 1. 
otorrinolaringológicas como corrección de estrabismo, adenotonsilectomías y miringotomías. Se ha descrito que el dolor es mayor en las primeras $24 \mathrm{~h}$, pero persiste en promedio 2 días, prolongándose hasta 9 días en caso de adenotonsilectomías[4]. Los procedimientos urológicos y de pared abdominal también destacan por sus altas tasas de dolor posoperatorio. En las primeras 24 h se han visto tasas importantes de dolor moderadosevero en circunsiciones (54\%), cistoscopías (25\%), reparación de hernia inguinal $(47 \%)$, orquidopexia (45\%) y reparación de hernia umbilical (67\%)[3].

\section{Técnicas de manejo del dolor}

\section{Analgesia sistémica}

AINES y paracetamol son comúnmente usados en niños, dado su perfil favorable de EA. Dado la incidencia de dolor severo descrita, algunos pacientes requieren opioides. Su perfil de EA como náuseas, vómitos o depresión respiratoria limitan su uso, especialmente en contexto ambulatorio dado que prolongan la estadía en recuperación y aumentan las consultas y hospitalizaciones no programadas[2],[7]. En USA se ha visto que un $4,8 \%$ de los niños (> 13 años) tienen un consumo persistente de opioides posterior a la cirugía, con riesgo de generar dependencia[8]. Por otro lado, se ha descrito mortalidad asociada a su uso en pacientes con trastornos obstructivos del sueño[9]. Considerando lo anterior, se han desarrollado protocolos ahorradores de opioides, donde una analgesia basada en AINES, dexmedetomidina, ketorolaco y bloqueos regionales proporcionan un manejo similar del dolor pero con menos EA y costos asociados[8].
Junto a la disminución del uso de opioides también se ha descrito un aumento del uso de paracetamol y ketorolaco en forma endovenosa. Esto es discutible ya que, al menos con el uso de paracetamol, las presentaciones endovenosas no son más efectivas que las orales y el costo es más elevado[2]. Con respecto a ketorolaco, éste es ampliamente usado en niños $(0,5$ $\mathrm{mg} / \mathrm{Kg} /$ dosis)[10] sin embargo, no está aprobado por la FDA. Una revisión sistemática Cochrane 2018, muestra que no hay evidencia suficiente para recomendar su uso, ni tampoco para evaluar su seguridad[11]

El celecoxib es un antiinflamatorio COX-2 selectivo ampliamente usado en adultos. En adenotonsilectomías, el uso de celecoxib (6 mg/kg, seguido de $3 \mathrm{mg} / \mathrm{kg}$ cada 12 h por 3 días) disminuyó el dolor en forma clínicamente significativa $(p=0,01)$ y el uso de paracetamol como analgesia de rescate $(78 \mathrm{mg} / \mathrm{kg}$, IC95\% 68-89 vs 97 mg/kg, IC95\% 85-109, p = 0,03), sin diferencias en EA, tasas de recuperación o satisfacción usuaria[12]. Parecoxib es otro antiinflamatorio COX-2 selectivo frecuentemente usado en adultos. En pacientes pediátricos estudios farmacocinéticos han demostrado que dosis de $1 \mathrm{mg} / \mathrm{kg}$, en $>$ de 2 años que pesan $10-70 \mathrm{~kg}$, son eficaces y seguras para tratar el dolor[13].

Respecto a otros AINES, al comparar la analgesia lograda en cirugía ortopédica con ibuprofeno $10 \mathrm{mg} / \mathrm{kg}$ versus morfina 0,5 $\mathrm{mg} / \mathrm{kg}$, no se han visto diferencias en manejo del dolor $(p=0,4)$ y necesidad de analgesia de rescate $(p=0,2)[14]$. Sin embargo, el uso de opioides se asocia a mayor incidencia de EA (69\% vs $39 \%, p<0,001)[14]$.

La Tabla 1 muestra dosis recomendadas y vías de administración de fármacos de uso común para analgesia sistémica perioperatoria:

\begin{tabular}{|c|c|c|}
\hline Fármaco & Vía de administración & Dosis \\
\hline \multicolumn{3}{|l|}{ AINES } \\
\hline \multirow[t]{2}{*}{ Ibuprofeno } & Rectal & $10 \mathrm{mg} / \mathrm{kg} \mathrm{c} / 8 \mathrm{~h}$ \\
\hline & Oral & $10 \mathrm{mg} / \mathrm{kg} \mathrm{c} / 8 \mathrm{~h}$ \\
\hline \multirow[t]{2}{*}{ Diclofenaco } & Rectal & $1 \mathrm{mg} / \mathrm{kg} \mathrm{c} / 8 \mathrm{~h}$ \\
\hline & Oral & $1 \mathrm{mg} / \mathrm{kg} \mathrm{c} / 8 \mathrm{~h}$ \\
\hline Naproxeno & Rectal & $5-7,5 \mathrm{mg} / \mathrm{kg} /$ dosis $\mathrm{c} / 12 \mathrm{~h}$ \\
\hline Ketorolaco & Endovenosa & 0,5 mg/kg (max $30 \mathrm{mg}$ ) dosis única; 0,15-0,2 mg/kg (max $10 \mathrm{mg}) \mathrm{c} / 6 \mathrm{~h}$ \\
\hline Ketoprofeno & Endovenosa & $1 \mathrm{mg} / \mathrm{kg} \mathrm{c} / 8 \mathrm{~h}$ \\
\hline \multirow[t]{3}{*}{ Paracetamol } & Rectal & $20-40$ mg/kg (15 mg/kg si < 10 kg), dosis única de carga junto a la anestesia \\
\hline & Oral & $15 \mathrm{mg} / \mathrm{kg} \mathrm{c/6-8} \mathrm{h}$ \\
\hline & Endovenosa & $\begin{array}{l}7,5 \mathrm{mg} / \mathrm{kg} \mathrm{si}<10 \mathrm{~kg} \\
15 \mathrm{mg} / \mathrm{kg} \mathrm{si}>10 \mathrm{~kg}\end{array}$ \\
\hline Metamizol & Endovenosa & $\begin{array}{l}10-15 \mathrm{mg} / \mathrm{kg} \mathrm{c} / 8 \text { h, 2,5 mg/kg/h infusión continua intraoperatoria a continuación de } \\
\text { dosis de carga }\end{array}$ \\
\hline \multicolumn{3}{|l|}{ Opioides } \\
\hline Fentanil & Endovenosa & 0,5-1 ug/kg uso postoperatorio, titular según efecto \\
\hline Morfina & Endovenosa & 25-100 ug/kg, titular según efecto \\
\hline Tramadol & Oral & 1-1,5 mg/kg c/4-6 h, titular según efecto (max 6 mg/kg/día) \\
\hline
\end{tabular}

Fuente: Adaptado de Vittinghoff et al[39]. 


\section{Analgesia regional}

Es considerada dentro de las mejores opciones analgésicas en cirugías ambulatorias[15]. En pediatría ha demostrado efectividad, seguridad, mejor satisfacción usuaria, menor consumo de opioides, menor estadía hospitalaria y menores tasas de dolor. El uso de ultrasonido (US) ha mejorado su perfil de seguridad y reducido las complicaciones asociadas. Los BC son ampliamente usados, sin embargo, las técnicas neuroaxiales se asocian a retención urinaria o bloqueo motor, lo que puede prolongar la estadía hospitalaria. Es por esto que los BNP resultan atractivos, ya que proporcionan similar analgesia evitando estas complicaciones[1],[5],[6].

Existen algunas consideraciones para su uso en pediatría:

- El consenso es la realización de bloqueos bajo anestesia general o sedación profunda por la necesidad de cooperación por parte del paciente.

- Se recomienda el uso de US para la realización de BNP, dado lo pequeño de los espacios y cercanía a estructuras vitales. Esto mejora la exactitud, eficacia y seguridad al compararlo con las técnicas estándar basadas en reparos anatómicos, que son más subjetivas.

- Las dosis de AL se basan en peso, existiendo consensos con dosis para cada tipo de bloqueo (Tabla 2).

\section{Técnicas de anestesia regional:}

- Bloqueo caudal (BC): Es de los más usados, siendo útil para procedimientos realizados infraumbilicales, como reparación de hernia inguinal, circuncisión y cirugías de extremidad inferior. Se ha utilizado en cirugías realizadas en región umbilical o supraumbilical, como hernioplastía umbilical, con un spread anestésico variable, lo que aumenta su tasa de fracaso[16]. Dentro de los riesgos está el bloqueo motor que impida la deambulación precoz. Se ha demostrado que bupivacaína, levobupivacaína y ropivacaína al $0,25 \%$ producen la misma analgesia posoperatoria, sin embargo, levobupivacaína y ropivacaína no han sido asociadas con esta complicación[1],[15]. El riesgo de retención urinaria es bajo, aproximadamente $2 \%[1],[15]$, siendo el mismo que el riesgo presentado en la anestesia general sin uso de $B C$. Dentro de sus contraindicaciones se encuentra: infección del sitio de punción, presencia de quistes pilonidales o disrafias. Las malformaciones espinales no contraindican el procedimiento si se realiza un estudio anatómico imagenológico[16].

- Bloqueo paravertebral: Es un bloqueo muy versátil ya que puede ser realizado en múltiples niveles, siendo una buena alternativa a la anestesia neuroaxial en procedimientos torácicos, abdominales o urológicos, especialmente los ambulatorios por permitir un alta más precoz. Puede ser realizado guiado por referencias anatómicas, neuroestimulación, pérdida de resistencia o US. Las complicaciones mayores son raras pero graves como neumotórax, punción intratecal, daño de nervios, hipotensión y cefalea[6]. La tasa de complicación es de 7\%, principalmente por falla del bloqueo[17]. Hay controversia en cuanto a dosis y número de inyecciones requeridas para ser efectivas, pero se ha visto que a nivel de T12-L1 la inyección única de 0,2-0,3 ml/kg puede cubrir hasta 6 dermátomos del abdomen inferior[5]. Se ha asociado con una mayor duración de la analgesia y menor necesidad de rescates analgésicos[6]. Puede además ser usado en forma bilateral. Representa una buena alternativa al bloqueo ilioinguinal/iliohipogástrico en hernia inguinal o hidrocele y una buena opción para orquidopexias, usándolo a nivel torácico bajo[15],[17].

- Bloqueo ilioinguinal/iliohipogástrico (IL/IH): Usado ampliamente en hernioplastías inguinales. Con uso limitado en cirugías testiculares, dado su innervación por raíces más altas (T10-L1)[15]. Ha demostrado ser equivalente al BC, con mínimos EA y complicaciones. Incluso puede proporcionar un mayor tiempo de analgesia y menos rescates analgésicos. Puede ser realizado guiándose por reparos anatómicos o ecografía. Con la primera técnica tiene una baja tasa de éxito (60\%). Dentro de sus complicaciones destaca la perforación de vísceras, inyección intravascular, parálisis de nervio femoral y parálisis del cuadriceps[6]. Con el uso de US las tasas de éxito mejoran, llegando a 94\%. La concentración de anestésico adecuada descrita es 0,25\%. El volumen de $\mathrm{AL}$ infundido debiera ser aproximadamente $0,1 \mathrm{ml} /$ $\mathrm{kg}$ con US, lo cual es menor que con técnica anatómica. El uso de volúmenes pequeños reduce el riesgo de bloqueo del nervio femoral por cercanía, lo cual es frecuente con el uso de la técnica por reparo anatómico (8,8\%)[5],[6[,[15].

\section{- Bloqueo del plano transverso abdominal (TAP block):} Bloqueo de planos interfasciales, cuyo uso principal es para proporcionar analgesia posoperatoria en cirugías abdominales o urológicas, comparable a las técnicas neuroaxiales pero sin los EA asociados[5],[6]. Sahin et al., demostró que su uso con dosis altas de AL proporciona mejor analgesia posoperatoria, disminuyendo el consumo de analgésicos de rescate en hernioplastía inguinal pediátrica[18]. En niños sometidos a cirugía abdominal baja unilateral, demostró un alta a domicilio más precoz $(306,8 \pm 18$ vs $259 \pm 22,4$ minutos, $p<0,001)$ y mayor satisfacción parental, sin diferencias en dolor, consumo de analgésicos y duración de la analgesia[19]. Se ha encontrado asociación, por metaanálisis, entre dosis de $A L$ y efecto en el dolor tardío y consumo de opioides en el posoperatorio, donde dosis mayores prolongan la duración y disminuyen la necesidad de analgesia de rescate en las primeras $24 \mathrm{~h}$. Dentro de las complicaciones está la perforación de vísceras, punción peritoneal e inyección intravascular, las que disminuyen con el uso de ecografía, reportandose en 0,3\% de los casos[5],[6],[17].

- Bloqueo de la vaina del recto: Se usa para proporcionar analgesia intra y posoperatoria en niños sometidos a incisiones de línea media abdominal o zonas periumbilicales, como también incisiones únicas a traves del ombligo. La técnica por reparos anatómicos es inexacta, por lo que se recomienda siempre usar US[5],[6],[17]. En reparación de hernia umbilical ha demostrado ser igual en términos de manejo del dolor al BC e infiltración de herida operatoria $(p<0,05)[20]$. Dentro de las complicaciones está la perforación de vísceras abdominales, inyección intravascular o hematoma retroperitoneal, las cuales se reducen con el uso de US[5],[6],[17].

- Bloqueo del cuadrado lumbar: Ha surgido como alternativa para cirugías abdominales altas o bajas. Existen cuatro aproximaciones para realizarlo, siendo el abordaje posterior el más seguro[21]. En pediatría, ha demostrado mejorar la analgesia posoperatoria disminuyendo la necesidad de analgesia de rescate y mejorando la satisfacción usuaria en 
Tabla 2. Dosis de anestésicos locales según tipo de bloqueo

\begin{tabular}{|c|c|c|}
\hline Tipo de bloqueo & Anestésico local & Dosis \\
\hline \multirow[t]{2}{*}{ Bloqueo caudal } & Bupivacaína 0,25\% o Ropivacaína 0,2\% & $\begin{array}{l}\text { Según dermatomos } \\
0,5 \mathrm{ml} / \mathrm{kg} \text { nivel sacro } \\
1 \mathrm{ml} / \mathrm{kg} \text { nivel lumbar } \\
1,25 \mathrm{ml} / \mathrm{kg} \text { nivel torácico }\end{array}$ \\
\hline & & $\begin{array}{l}\text { Dosis máxima } \\
\text { Bupivacaína/ L-Bupivacaína 2,5 mg/kg } \\
\text { Ropivacaína } 2 \mathrm{mg} / \mathrm{kg}\end{array}$ \\
\hline \multirow[t]{9}{*}{ Bloqueo ilioinguinal/iliohipogástrico } & & Técnica reparos anatómicos \\
\hline & Bupivacaína 0,25\% & $0,75-1,25 \mathrm{mg} / \mathrm{kg}(=0,3-0,5 \mathrm{ml} / \mathrm{kg})$ \\
\hline & L-Bupivacaína 0,25\% & $0,75-1,25 \mathrm{mg} / \mathrm{kg}(=0,3-0,5 \mathrm{ml} / \mathrm{kg})$ \\
\hline & Ropivacaína 0,2\% & $0,6-1 \mathrm{mg} / \mathrm{kg}(=0,3-0,5 \mathrm{ml} / \mathrm{kg})$ \\
\hline & & Guiado por ecografía \\
\hline & Bupivacaína 0,25\% & $0,1-0,2 \mathrm{ml} / \mathrm{kg}$ \\
\hline & & $0,1-0,2 \mathrm{ml} / \mathrm{kg}$ \\
\hline & L-Bupivacaína 0,25\% & $0,1-0,2 \mathrm{ml} / \mathrm{kg}$ \\
\hline & Ropivacaína 0,2\% & \\
\hline \multicolumn{3}{|l|}{ Bloqueo del nervio dorsal del pene } \\
\hline & Bupivacaína 0,25\% & $0,1-0,2 \mathrm{ml} / \mathrm{kg}$ \\
\hline & L-Bupivacaína 0,25\% & $0,1-0,2 \mathrm{ml} / \mathrm{kg}$ \\
\hline & Ropivacaína 0,2\% & $0,1-0,2 \mathrm{ml} / \mathrm{kg}$ \\
\hline \multicolumn{3}{|l|}{ Bloqueo recto abdominal/TAP block } \\
\hline & Bupivacaína 0,25\% & $0,2-0,5 \mathrm{ml} / \mathrm{kg}$ por lado \\
\hline & L-Bupivacaína 0,25\% & $0,2-0,5 \mathrm{ml} / \mathrm{kg}$ por lado \\
\hline & Ropivacaína 0,2\% & $0,2-0,5 \mathrm{ml} / \mathrm{kg}$ por lado \\
\hline \multicolumn{3}{|l|}{ Infiltración herida operatoria } \\
\hline & Bupivacaína 0,25\% & Dosis máxima 2,5 mg/kg (= $1 \mathrm{ml} / \mathrm{kg})$ \\
\hline & L-Bupivacaína 0,25\% & Dosis máxima 2,5mg/kg (= $1 \mathrm{ml} / \mathrm{kg})$ \\
\hline & Ropivacaína 0,2\% & Dosis máxima 3 mg/kg (= 1,5 ml/kg) \\
\hline \multicolumn{3}{|l|}{ Bloqueos continuos de nervio periférico } \\
\hline & L-Bupivacaína 0,125\% o Ropivacaína 0,2\% & Tasa de infusión 0,1-0,3mg/kg/h \\
\hline
\end{tabular}

Fuente: Adaptado de Vittinghoff et al[39], Lönnqvist et al[40].

comparación al TAP block, bloqueo IL/IH y BC en cirugías inguinales y orquidopexias[21],[22]. El spread anestésico que logra podría ser el responsable de estas diferencias, dado que logra aliviar tanto el dolor somático como visceral[21\}.

- Bloqueo del erector espinal: Descrito en 2016 por Forero, ha ganado amplia popularidad desde entonces. Al compararlo con el bloqueo IL/IH en cirugías inguinales, mejoró el manejo del dolor disminuyendo la necesidad de analgesia de rescate, y aumentó el tiempo de duración de la analgesia y la satisfacción parental, sin diferencias en efectos adversos[23]. Si bien faltan estudios randomizados que lo avalen y el mecanismo de acción no está del todo claro, constituye una buena alternativa analgésica en este tipo de cirugías, por su posible efecto en dolor visceral junto con proveer analgesia de pared abdominal[23].

- Bloqueo de nervio pudendo: En estudios observacionales de niños sometidos a circuncisión, reparación de hipospadia y otras cirugías peneanas, demostró no inferioridad comparado con $\mathrm{BC}$ en cuanto a analgesia posoperatoria. Como es más fácil de realizar y con mejor perfil de EA podría ser prefererido sobre éstos[24].

- Bloqueo de nervio dorsal del pene: Su uso en cirugía ambulatoria es frecuente en circunsiciones o cirugía de fimosis/parafimosis, donde ha demostrado efectividad superior a la analgesia tópica, sin embargo, se ha asociado a mayor tasa de complicaciones como hematomas[25].

- Bloqueos para cirugía otorrinolaringológica: En tonsilectomías se pueden realizar bloqueos locales en la fosa tonsilar o bloqueo del nervio glosofaríngeo. Las otoplastías se pueden manejar bloqueando el plexo cervical o con infiltración del sitio quirúrgico por parte del cirujano[14].

- Bloqueos para cirugía de cabeza y cuello: Son fáciles de hacer y seguros sin embargo, se realizan infrecuentemente. Estudios realizados en base a datos PRAN (Pediatric 
Regional Anesthesia Network) no han reportado secuelas o complicaciones importantes. El bloqueo del nervio auricular mayor es efectivo para cirugías de timpano-mastoide, donde ha demostrado reducir el consumo de opioides. También se puede realizar un bloqueo infraorbital, ideal para reparación de labio palatino, rinoplastías o cirugía endoscópica de senos paranasales. Una revisión Cochrane 2016 en fisura de labio palatino mostró que, si bien la evidencia es de baja calidad, sería superior al placebo para mejorar la analgesia. Para cirugía ocular el bloqueo peribulbar es muy usado, mostrando menor variabilidad hemodinámica, dolor postoperatorio, reflejo oculocardiaco y NVPO[1].

- Bloqueos de extremidades inferiores: Usados principalmente en cirugía ortopédica, siendo una alternativa a los BC. Sin embargo, aún falta evidencia de calidad que demuestre su eficacia y ventajas. En reparación de ligamento cruzado anterior, Lisgelia et al., demostraron que el bloqueo combinado femoral+ciático es mejor que el bloqueo femoral único y la infiltración intraarticular con bupivacaína, recibiendo menos opioides en el intraoperatorio $48 \%$ vs $100 \%$ vs $98 \%, p<0,0001)$ y posoperatorio $(28 \%$ vs $92 \%$ vs $96 \% \mathrm{p}<0,0001)$. Además, disminuyó scores de dolor $(1,5 \pm 2,0$ vs $4,5 \pm 2,0$ vs $2,8 \pm 1,8, p=0,0013)[26]$. Dado el riesgo de bloqueo motor de los bloqueos de nervio femoral o ciático, se han empezado a usar bloqueos más bajos y selectivos como bloqueo del canal obturador para cirugía de rodilla o nervio ciático o poplíteo para cirugía de pie[15],[17]. El riesgo de complicaciones generales ha sido descrito en torno al 1\%[16].

- Bloqueos de extremidades superiores: Se pueden realizar bloqueos de plexo braquial o infiltración local con anestésicos de larga duración, con poca evidencia disponible. Con respecto a los bloqueos de plexo braquial las técnicas supraclavicular o infraclavicular guiadas por ecografía son las más atractivas[15]. Las complicaciones más frecuentes son falla del bloqueo y analgesia intraoperatoria inadecua$\mathrm{da}$, con tasas alrededor del $2 \%$. Cuando se hacen bajo ecografía la tasa de complicaciones es prácticamente cero[17].

Bloqueos continuos de nervio periférico: Las técnicas únicas proveen analgesia por 8-12 h, lo cual es insuficiente en muchos casos. En cirugía ortopédica son cada vez más usados en niños, donde las tasas de dolor moderado-severo en domicilio son altas. No se han reportado complicaciones mayores (punciones vasculares, compromiso hemodinámico, toxicidad por $\mathrm{AL}$, daño neurológico, infecciones), siendo las complicaciones más frecuente las relacionadas a falla mecánica del catéter (8\%) como retiro accidental o filtración, con incidencias aproximadas de 1\% y 5\%[27]. Además, se han descrito tasas bajas de NVPO (5,9\%), buena analgesia y satisfacción usuaria[28]. Es requisito para su uso la adecuada educación de los cuidadores y el seguimiento diario por equipos especializados[1],[5],[17]. El uso de estos bloqueos permite mejorar el manejo domiciliario, disminuyendo el consumo de analgésicos orales, opioides y sus EA[29]. Se recomienda usar concentraciones bajas de $\mathrm{AL}$, para proveer analgesia evitando el bloqueo motor y reducir el riesgo de toxicidad por $\mathrm{AL}[17]$. Pareciera ser que pueden ser usados de forma segura con una educación y seguimiento adecuados, con uso de US y dosis bajas de AL[29] (Tabla 2).

\section{Adyuvantes}

Diversas drogas se han probado como adyuvantes a los bloqueos regionales, para extender o potenciar el efecto analgésico. La mejor evidencia está en $\mathrm{BC}$, con el uso de morfina, clonidina y ketamina[1],[15]. El uso de morfina no es opción en el contexto ambulatorio. Ketamina ha demostrado aumentar la duración y el efecto analgésico de concentraciones bajas de $\mathrm{AL}$ que por si solas no logran analgesia (sin adyuvante $291 \mathrm{~min}$; clonidina $2 \mathrm{mcg} / \mathrm{kg}$ : $492 \mathrm{~min}$; ketamina 0,5 mg/kg: $710 \mathrm{~min}$ ). En el contexto de bloqueos de nervio periférico solo la clonidina, en dosis de 1-2 ug/kg, ha demostrado aumentar la duración analgésica, en 20\%-50\%[5].

Dexametasona endovenosa ha demostrado aumentar la duración de los bloqueos regionales y reducir las NVPO[15]. En orquidopexia, su uso $(0,5 \mathrm{mg} / \mathrm{kg})$ posterior a la inducción anestésica sumado al BC con ropivacaína disminuyó el consumo de analgésicos de rescate (fentanil 7,9\% vs 38,5\%, p < 0,01; paracetamol 23,7 vs $64,1 \%, p<0,01)$ y aumentó el tiempo a la primera administración de paracetamol (646 vs 430 min, $p=$ 0,012 ). Además, mejoró scores de dolor posoperatorios, sin aumentar la incidencia de complicaciones[30]. En hernioplastías la administración de dexametasona 0,25 mg/kg endovenosa aumentó el tiempo hasta la primera dosis de analgesia de rescate $(654,18 \pm 31,56 \min$ vs $261,50 \pm 10,82 \mathrm{~min}, \mathrm{p}=0,0001) \mathrm{y}$ disminuyó el dolor significativamente $(p=0,0001)$. Además, disminuyó el consumo de paracetamol al alta $(38,30 \pm 17,95$ $\mathrm{mg}$ vs 55,30 $\pm 20,15 \mathrm{mg} p=0,0001)$. No se sabe exactamente como actúa pero se cree que es por su efecto antiinflamatorio[31].

Con respecto a la dexmedetomidina, la cual ha demostrado eficacia como adyuvante en adultos, un estudio randomizado en niños sometidos a hernioplastía con dosis de 0,3 ug/kg junto al $\mathrm{AL}$ en bloqueos IL/IH, prolongó el tiempo hasta la primera administración de analgesia de rescate $(4,0$ vs $7,6 h, p=0,0717)$ y disminuyó los scores de dolor (0 vs 7 pacientes con score $\geq$ $4, p=0,0029)[32]$. En cirugía abdominal baja, la adición de dexmedetomidina $2 \mathrm{ug} / \mathrm{kg}$ al $\mathrm{AL}$ aumentó el tiempo de analgesia postoperatoria (390 vs $750 \min p=0,001$ ) y disminuyó el consumo de analgésicos de rescate $(p<0,05)$, sin afectar la hemodinamia ni la duración del bloqueo motor (75 vs 90 p = 0,1349 ), con mayor duración de la sedación (30 vs 247,5 min p $=0,001)[33]$.

La ketamina ha demostrado prolongar la analgesia cuando se añade a los BC, sin embargo, se mantienen los riesgos de éstos. La instilación de AL en la herida operatoria es una técnica simple, de bajo costo y no invasiva que tiene la ventaja de tener un menor riesgo. En reparación de hernia inguinal se ha comparado el uso de ketamina como adyuvante al BC versus la instilación tópica en la herida operatoria de una solución de bupivacaína con ketamina. La administración tópica aumentó la duración de la analgesia $(28,74 \pm 2,88$ vs 21,43 $\pm 5,01$ h, $p=$ $0,000)$ y disminuyó los requerimientos de analgesia posoperatoria $(66,7 \%$ vs $87,5 \% ; p=0,01)$. Si bien se vio mayor delirium inicial, no hubo diferencias posteriormente y se registraron EA del BC como bloqueo motor o retención urinaria[34], sugieriendo que el uso tópico también puede ser una alternativa a los $\mathrm{BC}$.

Con respecto al uso de adyuvantes de forma sistémica la ketamina ha demostrado tener propiedades analgésicas, siendo cada vez más estudiada para este fin. En cirugía de estrabismo 
demostró que el uso intranasal comparada con paracetamol endovenoso tuvo mejor manejo del dolor ( $p>0,001)$, sin diferencias significativas en EA ( $p=0,94$ NVPO, $p=0,84$ mareos), aunque tuvo mayor sedación $(p<0,05)[35]$.

El uso de lidocaína tópica al 2\%-4\% en miringotomías ha demostrado reducir los requerimientos de analgésicos en el posoperatorio y proporcionar un alivio del dolor equivalente al paracetamol[36].

La dexmedetomidina también ha sido estudiada como adyuvante en forma endovenosa, con resultados discordantes. En niños sometidos a adenotonsilectomía el uso de dexmedetomidina $1 \mathrm{ug} / \mathrm{kg}$ posintubación proporcionó una analgesia similar a tramadol[37], sin embargo, con una mayor incidencia de EA como bradicardia e hipotensión intraoperatoria $(p=0,001)$ y mayor tiempo de extubación $(3,2 \pm 0,6$ min vs 6,8 $\pm 1,7$ min, $p=0,0012)$, sin diferencias significativas en el uso de morfina de rescate $(p=0,17)$ ni en las dosis de opioides usadas en el posoperatorio $(0,07 \pm 0,01 \mathrm{mg} / \mathrm{kg}$ vs $0,06 \pm 0,02 \mathrm{mg} / \mathrm{kg}, \mathrm{p}=$ $0,16)[37]$. Sin embargo, en otro estudio randomizado en niños con SAHOS sometidos a adenotonsilectomía, el uso de dexmedetomidina en dosis más altas, 2 ug/kg posterior a la inducción seguido de una infusión continua de 0,7 ug/ $\mathrm{kg} / \mathrm{h}$, comparado con fentanil, mostró menor necesidad de fentanil intraoperatorio $(9,8 \%$ vs $36 \%, p=0,001)$ y morfina de rescate en el posoperatorio (16,3\% vs 47,5\%, $p=.0,002)$, además, de reducir la incidencia $(p=0,028)$ y duración $(p=0,004)$ de episodios de agitación, sin eventos adversos hemodinámicos[38].

\section{Seguridad}

Numerosos estudios realizados con una muestra significativa de pacientes han mostrado la seguridad de la anestesia regional en niños. El último reportado en base a los datos PRAN, incluyó más de 50.000 bloqueos donde no se demostró un aumento de la mortalidad ni de las complicaciones[1].

El riesgo de complicaciones es bajo, menor al 1\%[1],[17],[39], sin embargo, en aquellos estudios que consideran la falla del bloqueo éste llega a $8 \%$, siendo ésta la complicación más frecuente[17]. No obstante, no se han reportado consecuencias graves como parálisis, intoxicación ni mortalidad.

La toxicidad por AL es infrecuente en niños, sin embargo, su detección es difícil especialmente en lactantes y neonatos por lo que la prevención es lo más importante. Ropivacaína y levobupivacaína han mostrado el mejor perfil de seguridad y debieran ser de elección cuando estén disponibles. Además, es importante el uso de dosis correctas y evitar condiciones que predispongan a la toxicidad como la hipoxemia, hipercarbia y acidosis. La aspiración previo a la inyección y el uso de US son fundamentales también para disminuir el riesgo de toxicidad[39].

Persisten algunas controversias con respecto a los BNP. En relación a la dosis segura, el test de epinefrina es difícil de realizar en niños porque los cambios hemodinámicos secundarios a la inyección intravascular de epinefrina no son iguales a los adultos, y por ende la ausencia de cambios hemodinámicos no descarta una inyección intravascular[1].

Por otro lado, se cree que los BNP podrían enmascarar el dolor isquémico de los síndromes compartimentales (SC), sin embargo, no hay evidencia de calidad que lo avale. De hecho, estudios sugieren que la presencia de dolor luego de un bloqueo bien realizado podría ser un indicador patognomónico de
SC. El diagnóstico tardío de SC podría ser más bien secundario a la dificultad en el reporte de síntomas de niños más pequeños. Las guías recomiendan usar concentraciones bajas de $\mathrm{AL}$ tanto en dosis única como infusión continua $(0,1 \%-0,25 \%$ y $0,1 \%$ respectivamente), usar juiciosamente los adyuvantes en cirugías de riesgo de compartimental y minimizar los volumenes de $A L[1]$.

\section{Conclusión}

Un número importante de pacientes pediátricos sufre dolor posoperatorio significativo. La cirugía ambulatoria será cada vez más frecuente por lo que se deben seguir desarrollando técnicas analgésicas seguras y efectivas, que permitan reducir costos y mejorar la calidad de la atención.

Gracias a la amplia disponibilidad de ultrasonido, la analgesia regional es cada vez más segura y eficaz, ofreciendo ventajas en el contexto de cirugía ambulatoria al permitir un alta precoz. Si bien se requieren más estudios para recomendar los BNP, la evidencia actual muestra bajas tasas de complicaciones[1], sin mortalidad asociada. Esto ha permitido generar protocolos libres de opioides, evitando los EA derivados de éstos y permitiendo manejar el dolor con analgésicos seguros como paracetamol o AINES, con altos grados de satisfacción usuaria. El dominio en estas técnicas por parte de los anestesiólogos es fundamental. La infiltración local también debe considerarse como complemento, ya que es una alternativa segura y fácil de realizar. Más investigación es necesaria con respecto a fármacos adyuvantes. El uso de catéteres para BNP continuos es factible cuando existe una educación a los cuidadores y seguimiento adecuado.

Para lograr una buena analgesia al alta suena razonable preferir AL de larga duración, sin embargo, se requieren más estudios para determinar las dosis máximas toleradas, asi como las diferencias farmacocinéticas y farmacodinámicas con adultos.

La creación de programas especializados en manejo del dolor traería beneficios, permitiendo un mejor seguimiento de los pacientes al alta y evitando las consultas inesperadas por dolor.

\section{Referencias}

1. Stein AL, Baumgard D, Del Rio I, Tutiven JL. Updates in Pediatric Regional Anesthesia and Its Role in the Treatment of Acute Pain in the Ambulatory Setting. Curr Pain Headache Rep. 2017 Feb;21(2):11. https://doi.org/10.1007/s11916-017-0614-z PMID:28251525

2. Rizeq YK, Many BT, Vacek JC, Silver I, Goldstein SD, Abdullah $\mathrm{F}$, et al. Trends in perioperative opioid and non-opioid utilization during ambulatory surgery in children. Surgery. 2019 Aug;166(2):172-6. https://doi.org/10.1016/j.surg.2019.04.005 PMID:31126588

3. Wilson CA, Sommerfield D, Drake-Brockman TF, Lagrange C, Ramgolam A, von Ungern-Sternberg BS. A prospective audit of pain profiles following general and urological surgery in children. Paediatr Anaesth. 2017 Nov;27(11):1155-64. https://doi. org/10.1111/pan.13256 PMID:29030932

4. Wilson CA, Sommerfield D, Drake-Brockman TF, von Bieberstein L, Ramgolam A, von Ungern-Sternberg BS. Pain after discharge 
following head and neck surgery in children. Paediatr Anaesth. 2016 Oct;26(10):992-1001. https://doi.org/10.1111/pan.12974 PMID:27397757

5. Visoiu M. Paediatric regional anaesthesia: a current perspective. Curr Opin Anaesthesiol. 2015 Oct;28(5):577-82. https://doi. org/10.1097/ACO.0000000000000238 PMID:26308515

6. Oliver JA, Oliver LA. Beyond the caudal: truncal blocks an alternative option for analgesia in pediatric surgical patients. Curr Opin Anaesthesiol. 2013 Dec;26(6):644-51. https://doi.org/10.1097/ AC0.0000000000000021 PMID:24126691

7. Jöhr M, Berger TM. Anaesthesia for the paediatric outpatient. Curr Opin Anaesthesiol. 2015 Dec;28(6):623-30. https://doi. org/10.1097/ACO.0000000000000246 PMID:26308518

8. Franz AM, Martin LD, Liston DE, Latham GJ, Richards MJ, Low DK. In Pursuit of an Opioid-Free Pediatric Ambulatory Surgery Center: A Quality Improvement Initiative. Anesth Analg; 2020. https://doi.org/10.1213/ANE.0000000000004774.

9. Lerman J. Pediatric ambulatory anesthesia: an update. Curr Opin Anaesthesiol. 2019 Dec;32(6):708-13. https://doi.org/10.1097/ ACO.0000000000000787 PMID:31503033

10. McLay JS, Engelhardt T, Mohammed BS, Cameron G, Cohen MN, Galinkin JL, et al. The pharmacokinetics of intravenous ketorolac in children aged 2 months to 16 years: A population analysis. Paediatr Anaesth. 2018 Feb;28(2):80-6. https://doi.org/10.1111/ pan.13302 PMID:29266539

11. McNicol ED, Rowe E, Cooper TE. Ketorolac for postoperative pain in children. Cochrane Database Syst Rev. 2018 Jul;7(7):CD012294. PMID:29981164

12. Murto K, Lamontagne C, McFaul C, MacCormick J, Ramakko KA, Aglipay $M$, et al. Celecoxib pharmacogenetics and pediatric adenotonsillectomy: a double-blinded randomized controlled study. Can J Anaesth. 2015 Jul;62(7):785-97. https://doi.org/10.1007/ s12630-015-0376-1 PMID:25846344

13. Hullett B, Salman S, O'Halloran SJ, Peirce D, Davies K, llett KF. Development of a population pharmacokinetic model for parecoxib and its active metabolite valdecoxib after parenteral parecoxib administration in children. Anesthesiology. 2012 May;116(5):1124-33. https://doi.org/10.1097/ ALN.0b013e31825154ef PMID:22450476

14. Poonai N, Datoo N, Ali S, Cashin M, Drendel AL, Zhu R, et al. Oral morphine versus ibuprofen administered at home for postoperative orthopedic pain in children: a randomized controlled trial. CMAJ. 2017 Oct;189(40):E1252-8. https://doi.org/10.1503/ cmaj.170017 PMID:29018084

15. Lönnqvist PA. Blocks for pain management in children undergoing ambulatory surgery. Curr Opin Anaesthesiol. 2011 Dec;24(6):627-32. https://doi.org/10.1097/ ACO.0b013e32834a276d PMID:21772142

16. Wiegele M, Marhofer $P$, Lönnqvist PA. Caudal epidural blocks in paediatric patients: a review and practical considerations. $\mathrm{Br}$ J Anaesth. 2019 Apr;122(4):509-17. https://doi.org/10.1016/j. bja.2018.11.030 PMID:30857607

17. Deer JD, Sawardekar A, Suresh S. Day surgery regional anesthesia in children: safety and improving outcomes, do they make a difference? Curr Opin Anaesthesiol. 2016 Dec;29(6):691-5. https:// doi.org/10.1097/AC0.0000000000000403 PMID:27685797

18. Sahin L, Sahin M, Gul R, Saricicek V, Isikay N. Ultrasoundguided transversus abdominis plane block in children: a randomised comparison with wound infiltration. Eur J Anaesthesiol. 2013 Jul;30(7):409-14. https://doi.org/10.1097/

\section{EJA.0b013e32835d2fcb PMID:23338056}

19. Elbahrawy K, El-Deeb A. Transversus abdomens plane block versus caudal block for postoperative pain control after daycase unilateral lower abdominal surgeries in children: A prospective, randomized study. Res Opin Anesth Intensive Care. 2016;3(1):20-4. https://doi.org/10.4103/2356-9115.184080.

20. Relland LM, Tobias JD, Martin D, Veneziano G, Beltran RJ, McKee $C$, et al. Ultrasound-guided rectus sheath block, caudal analgesia, or surgical site infiltration for pediatric umbilical herniorrhaphy: a prospective, double-blinded, randomized comparison of three regional anesthetic techniques. J Pain Res. 2017 Nov; 10:2629-34. https://doi.org/10.2147/JPR.S144259 PMID:29184439

21. Samerchua $A$, Leurcharusmee $P$, Panichpichate $K$, Bunchungmongkol N, Wanvoharn M, Tepmalai K, et al. A Prospective, randomized comparative study between ultrasound-guided posterior quadratus lumborum block and ultrasound-guided ilioinguinal/ iliohypogastric nerve block for pediatric inguinal herniotomy. Paediatr Anaesth. 2020 Apr;30(4):498-505. https://doi.org/10.1111/ pan.13837 PMID:32030845

22. Öksüz G, Arslan M, Urfalıoglu A, Güler AG, Teksen S, Bilal B, et al. Comparison of quadratus lumborum block and caudal block for postoperative analgesia in pediatric patients undergoing inguinal hernia repair and orchiopexy surgeries: a randomized controlled trial. Reg Anesth Pain Med. 2020 Mar;45(3):187-91. https://doi.org/10.1136/rapm-2019-101027 PMID:31907294

23. El-Emam EM, El Motlb EA. Ultrasound-Guided Erector Spinae versus Ilioinguinal/lliohypogastric Block for Postoperative Analgesia in Children Undergoing Inguinal Surgeries. Anesth Essays Res. 2019 Apr-Jun;13(2):274-9. https://doi.org/10.4103/aer. AER_81_19 PMID:31198244

24. Okoro C, Huang H, Cannon S, Low D, Liston DE, Richards MJ, et al. The pudendal nerve block for ambulatory urology: What's old is new again. A quality improvement project. J Pediatr Urol. 2020;16(5):594.e1-.e7.

25. Wang J, Zhao S, Luo L, Liu Y, Zhu Z, Li E, et al. Dorsal penile nerve block versus eutectic mixture of local anesthetics cream for pain relief in infants during circumcision: A meta-analysis. PLoS One. 2018 Sep;13(9):e0203439. https://doi.org/10.1371/journal. pone.0203439 PMID:30188927

26. Santana L, Lovejoy JF, Kiebzak G, Day J, Atanda A Jr, Mandel D. Comparison of Pain Scores and Medication Usage Between Three Pain Control Strategies for Pediatric Anterior Cruciate Ligament Surgery. Cureus. 2019 Aug;11(8):e5498. https://doi.org/10.7759/ cureus.5498 PMID:31511819

27. Visoiu M, Joy LN, Grudziak JS, Chelly JE. The effectiveness of ambulatory continuous peripheral nerve blocks for postoperative pain management in children and adolescents. Paediatr Anaesth. 2014 Nov;24(11):1141-8. https://doi.org/10.1111/pan.12518 PMID:25176318

28. Gable A, Burrier C, Stevens J, Wrona S, Klingele K, Bhalla T, et al. Home peripheral nerve catheters: the first 24 months of experience at a children's hospital. J Pain Res. 2016 Nov;9:1067-72. https://doi.org/10.2147/JPR.S110947 PMID:27920572

29. Simic D, Stevic M, Stankovic Z, Simic I, Ducic S, Petrov I, et al. The Safety and Efficacy of the Continuous Peripheral Nerve Block in Postoperative Analgesia of Pediatric Patients. Front Med (Lausanne). 2018 Mar;5:57. https://doi.org/10.3389/fmed.2018.00057 PMID:29594120

30. Hong JY, Han SW, Kim WO, Kim EJ, Kil HK. Effect of dexamethasone in combination with caudal analgesia on postoperative 
pain control in day-case paediatric orchiopexy. $\mathrm{Br} J$ Anaesth. 2010 Oct;105(4):506-10. https://doi.org/10.1093/bja/aeq187 PMID:20659915

31. Salami OF, Amanor-Boadu SD, Eyelade OR, Olateju SO. Effects of low-dose intravenous dexamethasone combined with caudal analgesia on post-herniotomy pain. Niger Postgrad Med J. 2017 Oct-Dec;24(4):230-5. https://doi.org/10.4103/npmj. npmj_120_17 PMID:29355162

32. Lundblad M, Marhofer D, Eksborg S, Lönnqvist PA. Dexmedetomidine as adjunct to ilioinguinal/iliohypogastric nerve blocks for pediatric inguinal hernia repair: an exploratory randomized controlled trial. Paediatr Anaesth. 2015 Sep;25(9):897-905. https:// doi.org/10.1111/pan.12704 PMID:26095747

33. Kamal M, Mohammed S, Meena S, Singariya G, Kumar R, Chauhan DS. Efficacy of dexmedetomidine as an adjuvant to ropivacaine in pediatric caudal epidural block. Saudi J Anaesth. 2016 OctDec;10(4):384-9. https://doi.org/10.4103/1658-354X.177325 PMID:27833479

34. Abdel-Ghaffar HS, Moeen SM, Moeen AM. Topical versus caudal ketamine/bupivacaine combination for postoperative analgesia in children undergoing inguinal herniotomy. Saudi J Anaesth. 2017 Jan-Mar;11(1):41-8. https://doi.org/10.4103/1658-354X.197338 PMID:28217052

35. Sanatkar M, Alireza ES, Takzare A. Analgesic effect of intranasal ketamine for the management of post-operative pain after strabismus surgery in pediatric patients. J Young Pharm. 2020;12(1):145-8. https://doi.org/10.5530/jyp.2020.12.29.

36. Robinson $\mathrm{H}$, Engelhardt $\mathrm{T}$. Ambulatory anesthetic care in chil- dren undergoing myringotomy and tube placement: current perspectives. Local Reg Anesth. 2017 Apr;10:41-9. https://doi. org/10.2147/LRA.S113591 PMID:28458577

37. Bedirli N, Akçabay M, Emik U. Tramadol vs dexmedetomidine for emergence agitation control in pediatric patients undergoing adenotonsillectomy with sevoflurane anesthesia: prospective randomized controlled clinical study. BMC Anesthesiol. 2017 Mar;17(1):41. https://doi.org/10.1186/s12871-017-0332-4 PMID:28283018

38. Patel A, Davidson M, Tran MC, Quraishi H, Schoenberg C, Sant $M$, et al. Dexmedetomidine infusion for analgesia and prevention of emergence agitation in children with obstructive sleep apnea syndrome undergoing tonsillectomy and adenoidectomy. Anesth Analg. 2010 Oct;111(4):1004-10. https://doi.org/10.1213/ ANE.0b013e3181ee82fa PMID:20705788

39. Vittinghoff $M$, Lönnqvist PA, Mossetti V, Heschl S, Simic D, Colovic $\mathrm{V}$, et al. Postoperative pain management in children: Guidance from the pain committee of the European Society for Paediatric Anaesthesiology (ESPA Pain Management Ladder Initiative). Paediatr Anaesth. 2018 Jun;28(6):493-506. https://doi. org/10.1111/pan.13373 PMID:29635764

40. Lönnqvist PA, Ecoffey C, Bosenberg A, Suresh S, Ivani G. The European society of regional anesthesia and pain therapy and the American society of regional anesthesia and pain medicine joint committee practice advisory on controversial topics in pediatric regional anesthesia I and II: what do they tell us? Curr Opin Anaesthesiol. 2017 Oct;30(5):613-20. https://doi.org/10.1097/ AC0.0000000000000508 PMID:28786855 